\title{
Positively curved Riemannian locally symmetric spaces are positively squared distance curved*
}

\author{
Philippe Delanoë and François Rouvière
}

\begin{abstract}
The squared distance curvature is a kind of two-point curvature the sign of which turned out crucial for the smoothness of optimal transportation maps on Riemannian manifolds. Positivity properties of that new curvature have been established recently for all the simply connected compact rank one symmetric spaces, except the Cayley plane. Direct proofs were given for the sphere, an indirect one (via the Hopf fibrations) for the complex and quaternionic projective spaces. Here, we present a direct proof of a property implying all the preceding ones, valid on every positively curved Riemannian locally symmetric space.
\end{abstract}

\section{Introduction}

A new notion of curvature was discovered in [MTW05] while investigating the smoothness of the solution of Monge's problem posed with smooth data (a problem briefly described below, see [Vil09]). The sign of a fourth order expression involving solely the cost function $c$ of the problem, a twopoint function, turned out crucial for the smoothness issue. The curvature nature of that expression (actually, of a push-forward of it, called cross-curvature) was elucidated in [KM10]. The expression itself was called cost-curvature [Loe09, Loe11] or $c$-curvature [DG10, DG] or else, up to a $\frac{3}{2}$ normalization factor, MTW tensor [FRV]. On a closed Riemannian manifold $M$ (closed meaning here: compact connected without boundary), the Brenier-McCann cost function, equal to (half) the distance $d$ squared, is an emblematic example for which Monge's problem with smooth data can be solved [Bre87, Bre91, McC01], in other words, for which one can find a measurable map $F: M \rightarrow M$ which pushes a given smooth positive probability measure $d \mu$ to another such one, and minimizes the total cost functional $\int_{M} \frac{1}{2} d^{2}(m, F(m)) d \mu$. In that case, the optimal map must read $F(m)=\exp _{m}(\nabla u(m))$ for some potential function $u: M \rightarrow \mathbb{R}$ and the $c$-curvature may be defined as follows:

*2000 MSC, Primary: 53C35; 53C21; Secondary: 53C26; 49N60; Keywords: symmetric spaces, rank one, positive curvature, almost-positive $c$-curvature

${ }^{\dagger}$ Supported by the CNRS at UNS 
Definition 1 (c-curvature) Let $m \in M$ and $V \in T_{m} M$ be such that the geodesic $t \in[0,1] \rightarrow \exp _{m}(t V)$ contains no cut point of $m$. For each $(X, Y) \in\left(T_{m} M\right)^{2}$, the c-curvature at $(m, V, X, Y)$ is given by:

$\mathcal{C}(m, V)(X, Y)=-\left.\frac{\partial^{4}}{\partial \tau^{2} \partial t^{2}} c\left(\exp _{m}(t X), \exp _{m}(V+\tau Y)\right)\right|_{t=0=\tau}$, with $c=\frac{1}{2} d^{2}$.

The smoothness of the optimal map $F$ is analyzed by viewing $F$ as a diffeomorphism whose potential function $u$ satisfies the Monge-Ampère equation which arises as the change of variable (or Jacobian) equation expressing the prescribed measure transport condition. The authors of [MTW05] were able to derive from that equation an interior one-sided a priori estimate on the second derivatives of $u$ based on the following condition which they denoted by (A3): there exists a constant $\sigma>0$ such that, for each $(m, V, X, Y)$ as in Definition 1, the lower bound $\mathcal{C}(m, V)(X, Y) \geqslant \sigma|X|^{2}|Y|^{2}$ holds provided $X$ and $Y$ are orthogonal. Assuming this condition forces the manifold $M$ to have its curvature bounded below by $\frac{3}{2} \sigma$ [Loe09] (see Remark 1 below). Neil Trudinger conjectured that a closed Riemannian manifold should satisfy (A3) provided it is positively curved with slowly varying Riemann tensor. The class of Riemannian locally symmetric spaces with positive curvature is thus a privileged one as regards the smoothness issue in optimal transportation theory. In [Loe11], condition (A3) was verified on the standard $n$-sphere and the desired smoothness result for the map $F$ followed from [Del04, DL06]. In order to deal with more general manifolds, variants of (A3) subsequently appeared, namely:

(i) the weaker condition (A3W) of [TW09] obtained by letting $\sigma=0$ in (A3), a condition shown to be necessary for the continuity of the optimal transport map [Loe09];

and two conditions due to Kim-McCann [KM] (see also [DG]):

(ii) the non-negatively $c$-curved (or NNCC) condition, obtained by dropping the condition $X \perp Y$ in (A3W);

(iii) the almost-positively c-curved (or APCC) condition, obtained from (NNCC) by further requiring that $\mathcal{C}(m, V)(X, Y)=0$ if and only if the span of the vectors $(V, X, Y)$ has dimension at most 1 .

The condition (NNCC) is stable under Riemannian products, unlike (A3W) if one of the factors does not satisfy (NNCC), and each of the above conditions is stable under Riemannian submersion $[\mathrm{KM}]$. Importantly, the condition (APCC) holds on $\mathbb{S}^{n}[\mathrm{KM}]$ (see also [Loe11, DG10, FR09]). Combining the latter result with the former stability one, applied to the Hopf fibrations $\mathbb{S}^{2 k+1} \rightarrow \mathbb{C} P^{k}$ [Bes78, Kli82] and $\mathbb{S}^{4 k+3} \rightarrow \mathbb{H} P^{k}$ [Bes78, GWZ86, Kar], Kim and McCann inferred that (A3) and (APCC) hold on $\mathbb{C} P^{k}$ and $\mathbb{H} P^{k}[\mathrm{KM}]$. 
Among simply connected compact rank one symmetric spaces, the sole case of $\mathbb{O} P^{2}$ remains open [Car26, Car27, Hel78, Kar]. The latter cannot be submersed by $\mathbb{S}^{23}$ with totally geodesic fibres (see [FIP04] and references therein). In the present note, we fill this gap by giving a direct proof of a somewhat stronger result, inspired from $[\mathrm{DG}]$, namely:

Theorem 1 Let $M$ be a closed Riemannian manifold. If $M$ is a positively curved Riemannian locally symmetric space, there exists a constant $\sigma>0$ such that, for each $(m, V) \in M \times T_{m} M$ as in Definition 1 and for every couple $(X, Y)$ of nonzero vectors of $T_{m} M$, setting $x=\frac{X}{|X|}, y=\frac{Y}{|Y|}$, the following inequality holds:

$$
\mathcal{C}(m, V)(X, Y) \geqslant \sigma \mathcal{A}_{2}(m, V, x, y)|X|^{2}|Y|^{2},
$$

where $\mathcal{A}_{2}(m, V, x, y)=1-\langle x, y\rangle^{2}+|V|^{2}-\langle x, V\rangle^{2}+|V|^{2}-\langle y, V\rangle^{2}$ is the sum of the squared areas of the parallelograms of $T_{m} M$ respectively defined by the couple of vectors $(x, y),(x, V),(y, V)$.

By the Cauchy-Schwarz inequality, $\mathcal{A}_{2}(m, V, x, y)$ is nonnegative, vanishing if and only if the span of $(V, x, y)$ has dimension at most 1 . Moreover, $\mathcal{A}_{2}(m, V, x, y) \geqslant 1$ if $x \perp y$. So Theorem 1 implies that the conditions (A3) and (APCC) hold on every closed positively curved Riemannian locally symmetric space. For convenience, let us call a manifold positively c-curved, or PCC, anytime it satisfies the conclusion of Theorem 1.

Remark 1 On every closed positively curved Riemannian manifold, the inequality (1) is obviously fulfilled along the zero section of $T M$, that is at $V=0$, due to the identity $\mathcal{C}(m, 0)(X, Y) \equiv \frac{2}{3} S_{m}(X, Y, X, Y)$, where $S_{m}$ denotes the sectional curvature tensor of $M$ at the point $m$ [Loe09, DG10]. Henceforth, we take $V \neq 0$ with no loss of generality.

Theorem 1 combined with the smoothness arguments given in [KM] yields the smoothness of the solution of Monge's problem with smooth data posed on an arbitrary simply connected positively curved Riemannian locally symmetric space. Dropping simple connectedness, such a smoothness result still holds by a standard covering space argument [DG10, p.412].

The outline of the paper is as follows. Section 2 contains a direct unified proof of Theorem 1 based on the validity of the latter for constant curvature spheres. The validity in question is established in Section 3. Finally, in Section 4, we enlarge the scope of Theorem 1 and point out an open question.

\section{Reduction of the proof to the case of constant curvature spheres}

In this section, we prove the following result: 
Proposition 1 If Theorem 1 holds for constant curvature spheres, it holds for any closed positively curved Riemannian locally symmetric space.

We will proceed by direct intrinsic calculations. Following Loeper's idea [Loe11], we will first compute a convenient expression of the Jacobi quadratic form which arises by taking the two $t$-derivatives of the cost function $c$ indicated in Definition 1. Then, we will be ready to differentiate that expression twice with respect to $\tau$ and obtain the desired $c$-curvature. Finally, taking it for granted on $\mathbb{S}^{n}[1]$, we will establish the lower bound (1) in the general case. Accordingly, the proof of Proposition 1 splits into three steps.

\subsection{Step 1: Jacobi quadratic form}

On a closed Riemannian manifold $M$, for each $(m, V)$ as in Definition 1 and $X \in T_{m} M$, the second derivative of the function $t \mapsto c\left(\exp _{m}(t X), \exp _{m}(V)\right)$ at $t=0$ is given by [DG10, FRV, Vil09]:

$$
\left.\frac{\partial^{2}}{\partial t^{2}} c\left(\exp _{m}(t X), \exp _{m}(V)\right)\right|_{t=0}=\left\langle\mathcal{J}_{V}(X), X\right\rangle
$$

where $\mathcal{J}_{V}$ is a symmetric endomorphism of $T_{m} M$ constructed as follows. Given $V \in T_{m} M$ such that the geodesic $t \in[0,1] \rightarrow \gamma(t)=\exp _{m}(t V)$ contains no conjugate point, the Jacobi equation along $\gamma$ (recalled below, see for instance [CE75, Kli82]) admits a unique solution $t \in[0,1] \rightarrow J(t) \in$ $T_{\gamma(t)} M$ with given Cauchy data $\left(J(0), \frac{\nabla J}{d t}(0)\right)$. Solutions of the Jacobi equation along $\gamma$ are called Jacobi fields along $\gamma$. The Jacobi endomorphism along $\gamma$ (or $V$ ) is the linear map $X \in T_{m} M \rightarrow \xi=\mathcal{J}_{V}(X) \in T_{m} M$ defined by demanding that the Jacobi field with Cauchy data $(X, 0)$ and the one with Cauchy data $(0, \xi)$ coincide at $t=1$.

Remark 2 The linear map, which associates to each vector $\xi \in T_{m} M$ the vector of $T_{\gamma(1)} M$ equal to the value at $t=1$ of the Jacobi field with Cauchy data $(0, \xi)$, is nothing but the map $d\left(\exp _{m}\right)(V): T_{m} M \rightarrow T_{\gamma(1)} M$. It is an isomorphism if and only if the geodesic $\gamma$ contains no conjugate point, as is well-known [CE75]. So, the latter condition on $\gamma$ (or else, on $(m, V)$ ) is exactly the one under which the Jacobi endomorphism $\mathcal{J}_{V}$ may be defined.

Let us compute the expression of $\mathcal{J}_{V}(X)$. The Jacobi equation along the geodesic $\gamma$ reads:

$$
\frac{\nabla^{2} J}{d t^{2}}+\mathcal{R}_{\dot{\gamma}(t)}(J)=0
$$

where $\dot{\gamma}=\frac{d \gamma}{d t}$ and $\mathcal{R}_{U}(\xi)=R_{p}(\xi, U) U$ whenever $p \in M,(U, \xi) \in\left(T_{p} M\right)^{2}$, setting $R_{p}$ for the Riemann tensor at $p$, here with the sign convention given by: $R_{p}(\xi, U)=\left[\nabla_{\xi}, \nabla_{U}\right]-\nabla_{[\xi, U]}[\mathrm{CE} 75, \mathrm{Kli} 82]$. For $U \neq 0$, the endomorphism $\mathcal{R}_{U}$ of $T_{p} M$ is symmetric and maps $U^{\perp}$ to itself. It is called the 
curvature operator along the vector $U$, and $\mathcal{R}_{\dot{\gamma}(t)}$, the curvature operator along the geodesic $\gamma$. From now on, we assume that $M$ is positively curved Riemannian locally symmetric.

Positive curvature implies that the eigenvalues of the curvature operator along any non zero vector $U \in T_{p} M$, restricted to $U^{\perp}$, are positive. The square root operator $\sqrt{\mathcal{R}_{U}}: T_{p} M \rightarrow T_{p} M$ is thus a well-defined symmetric endomorphism. Moreover, by local symmetry, the curvature operator is parallel along any geodesic, hence so is its square root. If $M$ is simply connected, it must be a symmetric space (Cartan's theorem) [CE75, KN63, KN69, Hel78] and each cut point must be a conjugate point [Cri62, Kar]. Furthermore, all the geodesics are simply closed of constant length [Cha70, Cha72, Kar], a length which we take equal to $\pi$ with no loss of generality. Now, for each $p \in M$ and non zero vector $U \in T_{p} M$, the eigenvalues of the operator $\sqrt{\mathcal{R}_{U}}$, restricted to $U^{\perp}$, are equal to $|U|$ or to $2|U|$ [Cha70, Cha72, Hel78, Kar]. Setting $E_{1}(U), E_{2}(U)$, for the corresponding eigenspaces and $E_{0}(U)=\mathbb{R} U$, we have the orthogonal decomposition:

$$
\forall p \in M, \forall U \in T_{p} M \backslash\{0\}, T_{p} M=E_{0}(U) \oplus E_{1}(U) \oplus E_{2}(U) .
$$

Besides, if $n=\operatorname{dim} M$ and $d=\operatorname{dim} E_{2}(U)$, then $d+1$ must divide $n$ [Cha70, Cha72] and be equal to one of the following values: $d=n-1$ when $M=$ $\mathbb{S}^{n}[4]$, or $d=1$ when $M=\mathbb{C} P^{k}$, or $d=3$ when $M=\mathbb{H} P^{k}$, or $d=7$ when $M=\mathbb{O} P^{2}$ [Car26, Car27, Hel78, Kar]. Here, with our normalization (length of simple closed geodesics equal to $\pi$ ), each of these spaces is understood to have the appropriate sectional curvature (holomorphic, quaternionic or octonionic) equal to 4.

Finally, if $M$ is not simply connected, the preceding properties hold for its universal covering space $\Pi: \widetilde{M} \rightarrow M$ equipped with the pulledback Riemannian metric. Since the covering map $\Pi$ is a local isometry, the spectral properties of the symmetric endomorphism $\sqrt{\mathcal{R}_{U}}: T M \rightarrow T M$ and the related eigenspaces decomposition (4) remain valid on $M$. In other words, the topology of $M$ is inessential for the calculation of the Jacobi endomorphism, hence for that of the $c$-curvature as well.

Using the splitting (4) with $(p, U)=(\gamma(t), \dot{\gamma}(t))$, for each $t \in[0,1]$, it is well-known [Bes78, p.82] that the Jacobi field $J$ along the geodesic $\gamma$ determined by the Cauchy data $(X, \xi) \in\left(T_{m} M\right)^{2}$ has the following expression:

$$
\begin{aligned}
& J(t)=\bar{X}_{0}(t)+t \bar{\xi}_{0}(t) \\
& +\cos (t|V|) \bar{X}_{1}(t)+\frac{\sin (t|V|)}{|V|} \bar{\xi}_{1}(t) \\
& +\cos (2 t|V|) \bar{X}_{2}(t)+\frac{\sin (2 t|V|)}{2|V|} \bar{\xi}_{2}(t),
\end{aligned}
$$

where we have denoted by $t \mapsto \bar{v}(t)$ the parallel vector field along $\gamma$ equal to $v \in T_{m} M$ at $t=0$. Indeed, one readily checks that $J(t)$, given by (5), 
satisfies (3) and that the Cauchy data $\left(J(0), \frac{\nabla J}{d t}(0)\right)$ coincide with $(X, \xi)$. Here, Remark 2 and our assumption on $V$ yield the important bound:

$$
2|V|<\pi \text {. }
$$

From (5) and (6), we infer that the Jacobi endomorphism reads:

$$
\mathcal{J}_{V}(X)=X_{0}+(|V| \cot |V|) X_{1}+(2|V| \cot 2|V|) X_{2},
$$

for every $X \in T_{m} M$, with $X_{i} \in E_{i}(V), i=0,1,2$. Note that, if $X_{1}=0$ (resp. $\left.X_{2}=0\right)$, this is formally the expression of the Jacobi endomorphism of $\mathbb{S}^{n}[1]$ (resp. $\mathbb{S}^{n}[4]$ ). Using the identity $2 \cot 2 r=\cot r-\tan r$ (with $r=|V|$ ), we can rewrite the right-hand side of the preceding equation as:

$$
\mathcal{J}_{V}(X)=X+(|V| \cot |V|-1)\left(X-X_{0}\right)-(|V| \tan |V|) X_{2} .
$$

Taking the scalar product with $X$, we obtain our first expression of the Jacobi quadratic form, namely:

(7) $\left\langle\mathcal{J}_{V}(X), X\right\rangle=|X|^{2}+(|V| \cot |V|-1)\left(|X|^{2}-\left|X_{0}\right|^{2}\right)-(|V| \tan |V|)\left|X_{2}\right|^{2}$.

Recalling (2) and anticipating the calculation of the $c$-curvature, we face a difficulty: if $X \neq 0$, the factors $\left|X_{i}\right|^{2}$ appearing on the right-hand side of (7) depend on $X$ and $V$; for fixed $X \neq 0$, how can we differentiate them with respect to $V$ ? The trick is to reverse the roles of $X$ and $V$ by considering the $2 \times 2$ system:

$$
\begin{gathered}
|V|^{2}\left|X_{1}\right|^{2}+|V|^{2}\left|X_{2}\right|^{2}=|V|^{2}|X|^{2}-|V|^{2}\left|X_{0}\right|^{2}, \\
|V|^{2}\left|X_{1}\right|^{2}+4|V|^{2}\left|X_{2}\right|^{2}=\left|\sqrt{\mathcal{R}_{V}}(X)\right|^{2},
\end{gathered}
$$

and by observing that the right-hand sides are symmetric with respect to $X$ and $V$. Indeed, on the one hand, from the very definition of $X_{0}$, we derive the equality $|V|^{2}\left|X_{0}\right|^{2}=\langle X, V\rangle^{2}$. On the other hand, from the symmetries of the Riemann tensor, we have $\left\langle\mathcal{R}_{V}(X), X\right\rangle \equiv\left\langle\mathcal{R}_{X}(V), V\right\rangle$, or else, $\left|\sqrt{\mathcal{R}_{V}}(X)\right|^{2} \equiv$ $\left|\sqrt{\mathcal{R}_{X}}(V)\right|^{2}$. In particular, we infer that

$$
|V|^{2}\left|X_{2}\right|^{2} \equiv|X|^{2}\left|V_{2}\right|^{2},
$$

where $V_{2} \in E_{2}(X)$ is given by the decomposition (4) arising from $X$. Thanks to Remark 1, we can plug in (7) the resulting expression of $\left|X_{2}\right|^{2}$, as well as that of $\left|X_{0}\right|^{2}$ just noted, getting:

$$
\begin{aligned}
\left\langle\mathcal{J}_{V}(X), X\right\rangle=\langle X, V\rangle^{2}\left(\frac{1-|V| \cot |V|}{|V|^{2}}\right)+|X|^{2} \mid & V|\cot | V \mid \\
& -\frac{\tan |V|}{|V|}|X|^{2}\left|V_{2}\right|^{2} .
\end{aligned}
$$

This is the expression of the Jacobi quadratic form which we will work with. 
Remark 3 If we let $V_{2}=0$ (resp. $V_{1}=0$ ) in (8), we obtain formally the expression of the Jacobi quadratic form of $\mathbb{S}^{n}[1]$ (resp. $\mathbb{S}^{n}[4]$ ). This is straightforward to see for $V_{2}=0$ (compare with [Loe11]) while, for $V_{1}=0$, it follows at once from the identity $\cot r-\tan r=2 \cot 2 r($ with $r=|V|)$.

\subsection{Step 2: c-curvature calculation}

We are ready to calculate $\mathcal{C}(m, V)(X, Y)$. We only have to do it when the length of $V$ lies in $\left(0, \frac{\pi}{2}\right)$ since, if $V=0$, we are done by Remark 1, while the bound $|V|<\frac{\pi}{2}$ comes from (6). Recalling Definition 1 and (2), we take $X \neq 0$ with no loss of generality, replace $V$ by $V+\tau Y$ in (8), and differentiate the resulting equation twice with respect to $\tau$ at $\tau=0$. After changing sign and using Remark 3 (with $V_{2}=0$ ), we obtain:

(9) $\mathcal{C}(m, V)(X, Y)=\mathcal{C}_{\mathbb{S}^{n}[1]}(m, V)(X, Y)$

$$
+|X|^{2} \frac{\partial^{2}}{\partial \tau^{2}}\left(\frac{\tan |V+\tau Y|}{|V+\tau Y|}\left|V_{2}+\tau Y_{2}\right|^{2}\right)_{\tau=0}
$$

where $\mathcal{C}_{\mathbb{S}^{n}[1]}(m, V)(X, Y)$ stands for the formal expression of the $c$-curvature which would arise on $\mathbb{S}^{n}[1]$ [DG10, p.106]. We defer the proof of (1) for constant curvature spheres to Section 3 and, as done in Proposition 1, we take it for granted in the sequel of this section.

\subsection{Step 3: proof of the lower bound (1)}

Suppose that we can prove the inequality:

$$
\frac{\partial^{2}}{\partial \tau^{2}}\left(\frac{\tan |V+\tau Y|}{|V+\tau Y|}\left|V_{2}+\tau Y_{2}\right|^{2}\right)_{\tau=0} \geqslant 0
$$

If so, the lower bound (1) follows from (9) since, by assumption, it holds for $\mathcal{C}_{\mathbb{S}^{n}[1]}(m, V)(X, Y)$. We are thus left with proving (10). Setting, for short, $r=|V|$ and $v=\frac{V}{r}$, a tedious calculation yields:

$$
\begin{aligned}
& \frac{\partial^{2}}{\partial \tau^{2}}\left(\frac{\tan |V+\tau Y|}{|V+\tau Y|}\left|V_{2}+\tau Y_{2}\right|^{2}\right)_{\tau=0}=2\left|Y_{2}\right|^{2} f(r) \\
& \quad+4\left\langle v_{2}, Y_{2}\right\rangle\langle v, Y\rangle g(r)+\left|v_{2}\right|^{2}\langle v, Y\rangle^{2} h(r)+\left|v_{2}\right|^{2}|Y|^{2} g(r)
\end{aligned}
$$

with:

$$
f(r)=\frac{\tan r}{r}, g(r)=\frac{1}{\cos ^{2} r}-f(r), h(r)=\frac{-3}{\cos ^{2} r}+3 f(r)+\frac{2 r \tan r}{\cos ^{2} r} .
$$

The functions $f, g, h$, are continuous on $r \in\left[0, \frac{\pi}{2}\right)$, respectively equal to $1,0,0$ at $r=0$, and one can readily check the lower bounds:

$$
\forall r \in\left[0, \frac{\pi}{2}\right), f(r) \geqslant 1 \text { and } g(r) \geqslant 0 .
$$


The lower bound on $g$ combined with the Cauchy-Schwarz inequality applied to $\left\langle v_{2}, Y_{2}\right\rangle$, yields:

$$
\begin{aligned}
\frac{\partial^{2}}{\partial \tau^{2}}\left(\frac{\tan |V+\tau Y|}{|V+\tau Y|}\left|V_{2}+\tau Y_{2}\right|^{2}\right)_{\tau=0} & \geqslant 2\left|Y_{2}\right|^{2} f(r) \\
& -4\left|Y_{2}\right|\left|v_{2}\right||\langle v, Y\rangle| g(r)+\left|v_{2}\right|^{2}\langle v, Y\rangle^{2} h(r) .
\end{aligned}
$$

Let us view the right-hand side of that inequality as a quadratic form $Q$ in the two variables $\left|Y_{2}\right|$ and $\left|v_{2}\right||\langle v, Y\rangle|$. The lower bound on $f$ implies the desired non negativity of $Q$, provided we can prove that the reduced discriminant of $Q$, equal to $D(r)=4 g^{2}(r)-2 f(r) h(r)$, satisfies $D<0$ on $r \in\left(0, \frac{\pi}{2}\right)$. Calculation yields:

$$
D(r)=-\frac{2 E(r)}{r^{2} \cos ^{3} r} \text { with } E(r)=\cos r(\sin r)^{2}+r \sin r-2 r^{2} \cos r .
$$

So the proof boils down to checking the inequality $E(r)>0$ on $\left(0, \frac{\pi}{2}\right)$. From a technical lemma of $[\mathrm{KM}]$ (used to prove that (APCC) holds on $\mathbb{S}^{n}[1]$ ), according to which: $\sin ^{2} r+r \sin r-r^{2}(1+\cos r)>0$ on $(0, \pi)$, we infer that $E(r)>r^{2} \cos r(1-\cos r)\left(\frac{\tan r}{r}-1\right)$ for every $r \in\left(0, \frac{\pi}{2}\right)$. The right-hand side of this inequality is clearly positive on $\left(0, \frac{\pi}{2}\right)$, so we are done.

Our proof of Proposition 1 implies that the conditions (A3) and (APCC) hold on any positively curved Riemannian locally symmetric space, since they are known to hold on $\mathbb{S}^{n}[1]$ [Loe11, DG10, FR09, KM].

\section{The case of constant curvature spheres}

Let us turn to the proof of

Proposition 2 Every constant curvature sphere is positively c-curved.

Before giving the proof itself, let us reduce it to the curvature 1 case. For each real $\kappa>0$, we set $\mathbb{S}^{n}[\kappa],\langle., .\rangle_{\kappa},|\cdot|_{\kappa}, d_{\kappa}, \mathcal{C}_{\kappa}(m, V)(X, Y)$, respectively for the sphere of curvature $\kappa$, its Riemannian metric, norm and distance function, and its $c$-curvature at $(m, V, X, Y)$ with $V$ satisfying:

$$
\sqrt{\kappa}|V|_{\kappa} \equiv|V|_{1}<\pi \text {. }
$$

We further denote by $\mathcal{B}_{\kappa}(m, V, X, Y)$ the expression $\mathcal{A}_{2}(m, V, x, y)|X|^{2}|Y|^{2}$ which occurs in the right-hand side of $(1)$ when calculated on $\mathbb{S}^{n}[\kappa]$. Note that the Levi-Civita connection of $\mathbb{S}^{n}[\kappa]$ is independent of the value of $\kappa>0$, hence so is the exponential map. Using this remark combined with the identity $d_{1} \equiv \sqrt{\kappa} d_{\kappa}$, we infer from Definition 1 the scaling relation:

$$
\mathcal{C}_{1}(m, V)(X, Y) \equiv \kappa \mathcal{C}_{\kappa}(m, V)(X, Y) .
$$


Besides, we readily get the pinching:

$$
\kappa^{p} \mathcal{B}_{\kappa}(m, V, X, Y) \geqslant \mathcal{B}_{1}(m, V, X, Y) \geqslant \kappa^{q} \mathcal{B}_{\kappa}(m, V, X, Y),
$$

with $(p, q)=(3,2)(\operatorname{resp} . \quad(p, q)=(2,3))$ if $\kappa>1$ (resp. if $\kappa \in(0,1))$. Combining the pinching with the scaling, we conclude that, for each $\kappa \neq 1$ :

(i) if (1) holds on $\mathbb{S}^{n}[1]$ with the constant $\sigma_{1}$, it must hold on $\mathbb{S}^{n}[\kappa]$ with the constant $\kappa^{q-1} \sigma_{1}$;

(ii) if (1) holds on $\mathbb{S}^{n}[\kappa]$ with the constant $\sigma_{\kappa}$, it must hold on $\mathbb{S}^{n}[1]$ with the constant $\kappa^{1-p} \sigma_{\kappa}$,

with $(p, q)$ given as above. So we have, indeed, reduced the proof of Proposition 2 to the curvature 1 case.

In $[\mathrm{DG}]$, the condition (PCC) was shown to hold on $\mathbb{S}^{2}[1]$; it remains to be proven only in higher dimension. To do so, let us revisit the proof of the (APCC) condition proposed for $\mathbb{S}^{n}[1]$ in the last section of $[\mathrm{KM}]$, focussing on its Step 1, where the calculation of $\mathcal{C}_{\mathbb{S}^{n}[1]}(m, V)(X, Y)$ is reduced to the 2-dimensional case. Given $(m, V) \in T \mathbb{S}^{n}$ satisfying (11), we assume with no loss of generality that $V, X$ and $Y$ do not vanish and set, for short:

$$
x=\frac{X}{|X|}, y=\frac{Y}{|Y|} \text { and still } r=|V|, v=\frac{V}{r} .
$$

If $v$ is parallel to $y$, it was shown in [KM] that:

$$
\mathcal{C}_{\mathbb{S}^{n}[1]}(m, V)(X, Y)=2 c_{3}(r)\left(1-\langle x, v\rangle^{2}\right)|X|^{2}|Y|^{2},
$$

where $c_{3}(r)=\frac{1-r \cot r}{\sin ^{2} r}$ is the function so denoted, and shown bounded below by $\frac{1}{3}$ on $[0, \pi)$, in [FR09, p.1701]. In that case, we infer that (1) holds for $\mathcal{C}_{\mathbb{S}^{n}[1]}(m, V)(X, Y)$ with $\sigma=\frac{2}{3\left(1+\pi^{2}\right)}$. If $v$ is not parallel to $y$, setting $\mathfrak{p}$ for the orthogonal projection of $T_{m} \mathbb{S}^{n}$ on the 2-plane spanned at $m$ by $(v, y)$, it was shown in $[\mathrm{KM}]$ that:

$$
\begin{aligned}
\mathcal{C}_{\mathbb{S}^{n}[1]}(m, V)(X, Y) & =\mathcal{C}_{\mathbb{S}^{2}[1]}(m, V)(\mathfrak{p} X, Y) \\
& +\left(2 c_{3}(r)\langle y, v\rangle^{2}+c_{5}(r)\left(1-\langle y, v\rangle^{2}\right)\right)|X-\mathfrak{p} X|^{2}|Y|^{2},
\end{aligned}
$$

where $c_{5}(r)=\frac{1}{\sin ^{2} r}-\frac{\cot r}{r}$ is the function so denoted, and shown bounded below by $\frac{2}{3}$ on $[0, \pi)$, in [FR09, p.1701]. Therefore, we obtain:

$$
\mathcal{C}_{\mathbb{S}^{n}[1]}(m, V)(X, Y) \geqslant \mathcal{C}_{\mathbb{S}^{2}[1]}(m, V)(\mathfrak{p} X, Y)+\frac{2}{3}|X-\mathfrak{p} X|^{2}|Y|^{2} .
$$


If $\mathfrak{p} X=0$, we get at once (1) with $\sigma=\frac{2}{3\left(1+2 \pi^{2}\right)}$. If $\mathfrak{p} X \neq 0$, we recall from $[\mathrm{DG}]$ the existence of a constant $\alpha>0$ such that:

$$
\mathcal{C}_{\mathbb{S}^{2}[1]}(m, V)(\mathfrak{p} X, Y) \geqslant \alpha \mathcal{B}_{1}(m, V, \mathfrak{p} X, Y)
$$

and note the obvious lower bound:

$$
\begin{aligned}
\frac{2}{3}|X-\mathfrak{p} X|^{2}|Y|^{2} \geqslant \frac{2}{9}|X-\mathfrak{p} X|^{2}|Y|^{2} & +\frac{2}{9 \pi^{2}}|X-\mathfrak{p} X|^{2}|Y|^{2}|V|^{2} \\
& +\frac{2}{9 \pi^{2}}|X-\mathfrak{p} X|^{2}\left(|Y|^{2}|V|^{2}-\langle Y, V\rangle^{2}\right)
\end{aligned}
$$

Plugging these inequalities in (12) yields (1) with $\sigma=\min \left(\alpha, \frac{2}{9 \pi^{2}}\right)$. Altogether, the lower bound (1) thus holds for $\mathcal{C}_{\mathbb{S}^{n}[1]}(m, V)(X, Y)$ with the latter choice of $\sigma$, uniformly with respect to $(m, V, X, Y)$ with $|V|<\pi$.

\section{Conclusion}

We would like to conclude this note by giving its full scope to Theorem 1. We require a more general definition of the $c$-curvature used in [FRV]. Recalling Remark 2, it can be stated as follows:

Definition 2 (extended $c$-curvature) Let $M$ be a complete Riemannian manifold and $(m, V) \in T M$ such that the geodesic $t \in[0,1] \rightarrow \exp _{m}(t V) \in$ $M$ contains no conjugate point. Set $\mathcal{J}_{V}$ for the corresponding Jacobi endomorphism of $T_{m} M$. For each $(X, Y) \in\left(T_{m} M\right)^{2}$, the extended c-curvature at $(m, V, X, Y)$ is given by: $\overline{\mathcal{C}}(m, V)(X, Y)=-\left.\frac{\partial^{2}}{\partial \tau^{2}}\left\langle\mathcal{J}_{V+\tau Y}(X), X\right\rangle\right|_{\tau=0}$.

The difference between our two definitions of the $c$-curvature lies in the assumption made on $(m, V)$, which is stronger in Definition 1 . Under the assumption of Definition 1, from (2), we know that $\overline{\mathcal{C}}(m, V) \equiv \mathcal{C}(m, V)$. In particular, back to $M$ positively curved Riemannian locally symmetric, the two definitions coincide anytime $M$ is simply connected because, then, each cut point is a conjugate point [Cri62]. But Definition 2 is more general than Definition 1 if $M$ has non trivial topology. In that case, the proof given in this note shows that the lower bound (1) remains valid for $\overline{\mathcal{C}}(m, V)(X, Y)$. In other words, Theorem 1 holds for the extended $c$-curvature.

Finally, the result of this paper prompts us toward a stability question in the spirit of the Trudinger's conjecture mentioned in the introduction, namely: is every closed positively curved Riemannian manifold, positively $c$-curved, provided the gradient of its Riemann tensor is small enough in $C^{1}$ norm ? This is a difficult question, settled (affirmatively) only on $\mathbb{S}^{2}[\mathrm{DG}]$. 


\section{References}

[Bes78] Arthur L. Besse. Manifolds all of whose geodesics are closed, volume 93 of Ergebnisse der Mathematik und ihrer Grenzgebiete [Results in Mathematics and Related Areas]. Springer-Verlag, Berlin, 1978. With appendices by D. B. A. Epstein, J.-P. Bourguignon, L. Bérard-Bergery, M. Berger and J. L. Kazdan.

[Bre87] Yann Brenier. Décomposition polaire et réarrangement monotone des champs de vecteurs. C. R. Acad. Sci. Paris Sér. I Math., 305(19):805-808, 1987.

[Bre91] Yann Brenier. Polar factorization and monotone rearrangement of vector-valued functions. Comm. Pure Appl. Math., 44(4):375417, 1991.

[Car26] E. Cartan. Sur une classe remarquable d'espaces de Riemann. Bull. Soc. Math. France, 54:214-264, 1926.

[Car27] E. Cartan. Sur une classe remarquable d'espaces de Riemann. II. Bull. Soc. Math. France, 55:114-134, 1927.

[CE75] Jeff Cheeger and David G. Ebin. Comparison theorems in Riemannian geometry. North-Holland Publishing Co., Amsterdam, 1975. North-Holland Mathematical Library, Vol. 9.

[Cha70] Isaac Chavel. On Riemannian symmetric spaces of rank one. Advances in Math., 4:236-263 (1970), 1970.

[Cha72] Isaac Chavel. Riemannian symmetric spaces of rank one. Marcel Dekker Inc., New York, 1972. Lecture Notes in Pure and Applied Mathematics, Vol. 5.

[Cri62] Richard J. Crittenden. Minimum and conjugate points in symmetric spaces. Canad. J. Math., 14:320-328, 1962.

[Del04] Ph. Delanoë. Gradient rearrangement for diffeomorphisms of a compact manifold. Differential Geom. Appl., 20(2):145-165, 2004 .

[DG] Philippe Delanoë and Yuxin Ge. Locally nearly spherical surfaces are almost-positively c-curved. Methods Appl. Anal. Preprint 2010, accepted August 2011.

[DG10] Philippe Delanoë and Yuxin Ge. Regularity of optimal transport on compact, locally nearly spherical, manifolds. J. Reine Angew. Math., 646:65-115, 2010. 
[DL06] Philippe Delanoë and Grégoire Loeper. Gradient estimates for potentials of invertible gradient-mappings on the sphere. Calc. Var. Partial Differential Equations, 26(3):297-311, 2006.

[FIP04] Maria Falcitelli, Stere Ianus, and Anna Maria Pastore. Riemannian submersions and related topics. World Scientific Publishing Co. Inc., River Edge, NJ, 2004.

[FR09] Alessio Figalli and Ludovic Rifford. Continuity of optimal transport maps and convexity of injectivity domains on small deformations of $\mathbb{S}^{2}$. Comm. Pure Appl. Math., 62(12):1670-1706, 2009.

[FRV] Alessio Figalli, Ludovic Rifford, and Cédric Villani. Nearly round spheres look convex. American J. Math. Preprint 2009, accepted April 2011.

[GWZ86] Herman Gluck, Frank Warner, and Wolfgang Ziller. The geometry of the Hopf fibrations. Enseign. Math. (2), 32(3-4):173-198, 1986.

[Hel78] Sigurdur Helgason. Differential geometry, Lie groups, and symmetric spaces, volume 80 of Pure and Applied Mathematics. Academic Press Inc. [Harcourt Brace Jovanovich Publishers], New York, 1978.

[Kar] Hermann Karcher. A geometric classification of positively curved symmetric spaces and the isoparametric construction of the Cayley plane. "On the geometry of differentiable manifolds", Workshop, Rome 1986, Société Mathématique de France, Astérisque 163/164, 111-135, 1988.

[Kli82] Wilhelm Klingenberg. Riemannian geometry, volume 1 of de Gruyter Studies in Mathematics. Walter de Gruyter \& Co., Berlin, 1982.

[KM] Young-Heon Kim and Robert J. McCann. Towards the smoothness of optimal maps on Riemannian submersions and Riemannian products (of round spheres in particular). J. Reine Angew. Math. Preprint 2008, accepted January 2010.

[KM10] Young-Heon Kim and Robert J. McCann. Continuity, curvature, and the general covariance of optimal transportation. J. Eur. Math. Soc. (JEMS), 12(4):1009-1040, 2010.

[KN63] Shoshichi Kobayashi and Katsumi Nomizu. Foundations of differential geometry. Vol I. Interscience Publishers, a division of John Wiley \& Sons, New York-Lond on, 1963. 
[KN69] Shoshichi Kobayashi and Katsumi Nomizu. Foundations of differential geometry. Vol. II. Interscience Tracts in Pure and Applied Mathematics, No. 15 Vol. II. Interscience Publishers John Wiley \& Sons, Inc., New York-London-Sydney, 1969.

[Loe09] Grégoire Loeper. On the regularity of solutions of optimal transportation problems. Acta Math., 202(2):241-283, 2009.

[Loe11] Grégoire Loeper. Regularity of optimal maps on the sphere: the quadratic cost and the reflector antenna. Arch. Ration. Mech. Anal., 199(1):269-289, 2011.

[McC01] Robert J. McCann. Polar factorization of maps on Riemannian manifolds. Geom. Funct. Anal., 11(3):589-608, 2001.

[MTW05] Xi-Nan Ma, Neil S. Trudinger, and Xu-Jia Wang. Regularity of potential functions of the optimal transportation problem. Arch. Ration. Mech. Anal., 177(2):151-183, 2005.

[TW09] Neil S. Trudinger and Xu-Jia Wang. On the second boundary value problem for Monge-Ampère type equations and optimal transportation. Ann. Sc. Norm. Super. Pisa Cl. Sci. (5), 8(1):143-174, 2009.

[Vil09] Cédric Villani. Optimal transport. Old and new, volume 338 of Grundlehren der Mathematischen Wissenschaften [Fundamental Principles of Mathematical Sciences]. Springer-Verlag, Berlin, 2009.

Université de Nice-Sophia Antipolis Laboratoire J.-A. Dieudonné, Parc Valrose F-06108 Nice Cedex 2 E-mails: Philippe.Delanoe@unice.fr, Francois.Rouviere@unice.fr 